\title{
Density Forecasting for Long-Term Peak Electricity Demand
}

\author{
Rob J. Hyndman and Shu Fan, Member, IEEE
}

\begin{abstract}
Long-term electricity demand forecasting plays an important role in planning for future generation facilities and transmission augmentation. In a long-term context, planners must adopt a probabilistic view of potential peak demand levels. Therefore density forecasts (providing estimates of the full probability distributions of the possible future values of the demand) are more helpful than point forecasts, and are necessary for utilities to evaluate and hedge the financial risk accrued by demand variability and forecasting uncertainty. This paper proposes a new methodology to forecast the density of long-term peak electricity demand.

Peak electricity demand in a given season is subject to a range of uncertainties, including underlying population growth, changing technology, economic conditions, prevailing weather conditions (and the timing of those conditions), as well as the general randomness inherent in individual usage. It is also subject to some known calendar effects due to the time of day, day of week, time of year, and public holidays.

A comprehensive forecasting solution is described in this paper. First, semi-parametric additive models are used to estimate the relationships between demand and the driver variables, including temperatures, calendar effects and some demographic and economic variables. Then the demand distributions are forecasted by using a mixture of temperature simulation, assumed future economic scenarios, and residual bootstrapping. The temperature simulation is implemented through a new seasonal bootstrapping method with variable blocks.

The proposed methodology has been used to forecast the probability distribution of annual and weekly peak electricity demand for South Australia since 2007. The performance of the methodology is evaluated by comparing the forecast results with the actual demand of the summer 2007-2008.
\end{abstract}

Index Terms-Density forecast, long-term demand forecasting, simulation, time series.

\section{INTRODUCTION}

$\mathbf{E}$ LECTRICITY demand forecasting is a key task for the effective operation and planning of power systems. Demand forecasting is concerned with the prediction of hourly, daily, weekly, and annual values of the system demand and peak demand. Such forecasts are sometimes categorized as short-term, medium-term and long-term forecasts, depending on the time horizon.

Manuscript received August 07, 2008; revised September 02, 2009. Paper no. TPWRS-00653-2008.

The authors are with the Business and Economic Forecasting Unit, Monash University, Clayton, VIC 3800, Australia (e-mail: Rob.Hyndman@buseco.monash.edu.au; Shu.Fan@buseco.monash.edu.au).

Color versions of one or more of the figures in this paper are available online at http://ieeexplore.ieee.org.

Digital Object Identifier 10.1109/TPWRS.2009.2036017
Long-term demand forecasting, usually corresponding to the forecast horizon from several months to several years ahead, is an integral process in scheduling the construction of new generation facilities and in the development of transmission and distribution systems. An overestimate of long-term electricity demand will result in substantial wasted investment in the construction of excess power facilities, while an underestimate of demand will result in insufficient generation and unmet demand.

In the literature to date, short-term demand forecasting has attracted substantial attention due to its importance for power system control, unit commitment, economic dispatch and electricity markets. On the other hand, medium- and long-term forecasting have not received as much attention, despite their value for system planning and budget allocation.

The natures of long-term and short-term demand forecasts are quite different. For short-term forecasting, people are often interested in point forecasts (i.e., forecasts of the mean or median of the future demand distribution). For long-term forecasting, point forecasts are of limited interest as they cannot be used to evaluate and hedge the financial risk accrued by demand variability and forecasting uncertainty. Instead, density forecasts (providing estimates of the full probability distributions of the possible future values of the demand) are more helpful and necessary for long-term planning. For example, the National Electricity Market (NEM) of Australia asks for different levels of the probability of exceedance (POE) to be provided for all long-term forecasts [1]. Another difference between short-term and long-term demand forecasting is in their use of meteorological information. It is well known that meteorological variables are the key inputs for most demand models. For short-term forecasts (up to one week ahead), such information can be obtained from weather services; but it is unavailable for long-term forecasts and so a feasible method to generate realistic future temperatures is required.

A wide variety of forecasting techniques have been proposed in the past few years, mostly assuming short-term forecast horizons. These techniques can be classified into several groups: statistical models including linear regression and time series methods [2]-[6]; artificial intelligent approaches including artificial neural networks and fuzzy logic methods [7]-[12]; and other methods including machine learning, data mining techniques and grey models [13]-[15]. For long-term demand forecast horizons, a few researchers have proposed interval and probabilistic forecasts, notably [6] and [15]. In this paper, a comprehensive methodology to forecast the density of annual and weekly peak electricity demand up to ten years ahead is proposed based on this latter work.

Peak electricity demand in a given season is subject to a range of uncertainties, including underlying population growth, 
changing technology, economic conditions, prevailing weather conditions (and the timing of those conditions), as well as the general randomness inherent in individual usage. To deal with the various uncertainties and obtain density forecasts, the relationships between demand and the driver variables are estimated using semi-parametric additive models. Then the demand distributions are forecasted using a mixture of temperature simulation, assumed future economic scenarios, and residual bootstrapping. The temperature simulation is implemented through a new seasonal bootstrapping method with variable blocks.

The proposed methodology has been applied to forecast the probability distributions of annual and weekly peak electricity demand up to ten years ahead for South Australia since 2007. The ten-year probability of exceedance values (conditional on predicted economic and demographic variables) for various probabilities including $10 \%, 50 \%$ and $90 \%$ have been calculated in this paper. To evaluate the performance of the proposed methodology, the actual and forecast results for each summer are computed. The ten-year forecasts based on data observed to the end of the summer of 2007-2008 are also computed. The results show good forecasting capacity of the proposed methodology at predicting the forecast distribution.

\section{DATA DESCRIPTION AND ANALYSIS}

\section{A. Load and Temperature Data}

The forecasts presented in this paper are for South Australian "native demand", being the demand met by both scheduled and nonscheduled generators supplying the South Australian region of the NEM. Each day is divided into 48 half-hourly periods which correspond with NEM settlement periods.

South Australia is Australia's fourth largest state and is largely a desert region. The population of about 1.6 million is heavily concentrated in the capital city, Adelaide, and in nearby areas. Half-hourly demand data were obtained from Australian Energy Market Operator (AEMO) from July 1, 1997 to March 31, 2008.

Because our interest is in peak electricity demand, which occurs without exception in summer for South Australia, only data from November-March were retained for analysis and modeling. All data from April-October for each year were omitted. Thus, each "year" consists of 151 days. Here, the period November-March is defined as "summer" for the purposes of this paper.

Time plots of the half-hourly demand data are illustrated in Figs. 1 and 2, which clearly show the intra-day pattern (of length 48 ) and the weekly seasonality (of length $48 \times 7=336$ ); the annual seasonality (of length $48 \times 151=7248$ ) is less obvious.

The demand values include relatively large major industrial loads (from mining companies in South Australia). The halfhourly major industrial demand is given in Fig. 3. It can be seen that this load has increased from an average of around $50 \mathrm{MW}$ to more than double, and is likely to grow strongly in the future. Although this load can vary considerably over time (e.g., plant outages at the sites that have sometimes persisted for several months), the load is not temperature sensitive. Therefore, it will be considered separately.

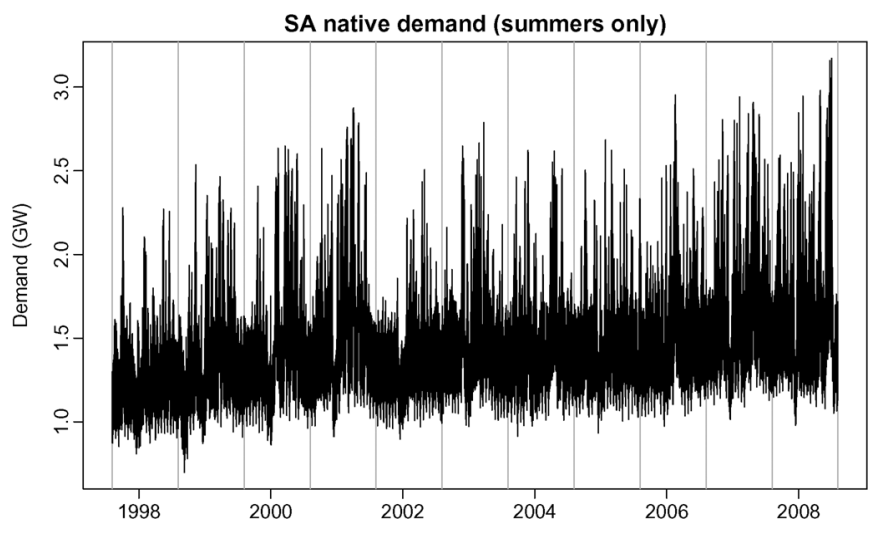

Fig. 1. Half-hourly demand data for South Australia from July 1, 1997 to March 31, 2008. Only data from November-March are shown.

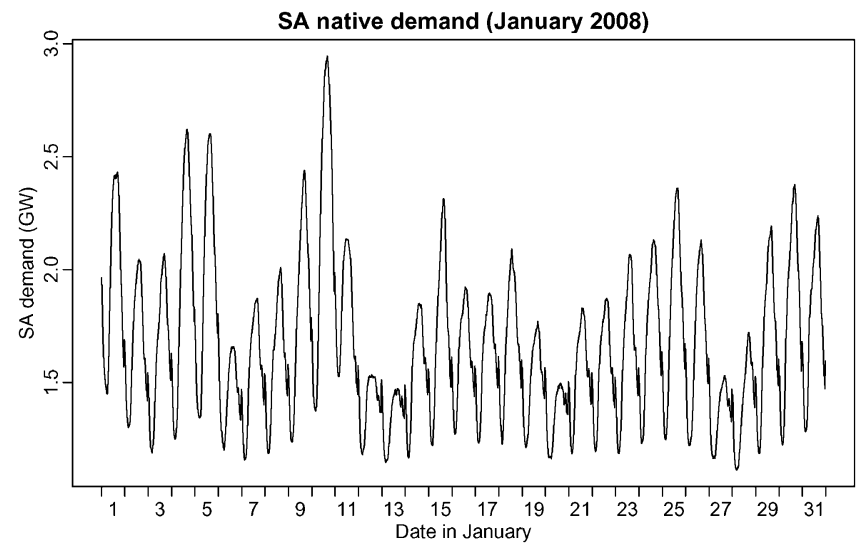

Fig. 2. Half-hourly demand data for South Australia, January 2008.

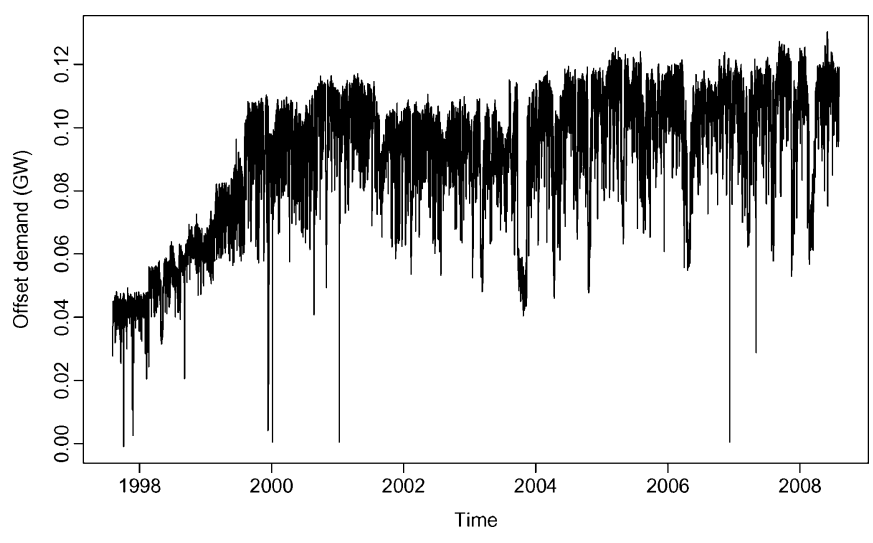

Fig. 3. Half-hourly demand data for major industries. July 1, 1997-March 31, 2008.

Half-hourly temperature data for two different locations in high demand areas (Kent Town and Adelaide Airport) have been considered in this paper. The relationship between demand (excluding major industrial loads) and average temperature is shown in Fig. 4, where a nonlinear relationship between load and temperature can be observed.

\section{B. Demographic and Economic Data}

The long-term electricity demand growth is largely dependent on demographic and economic variables. In this paper, the 


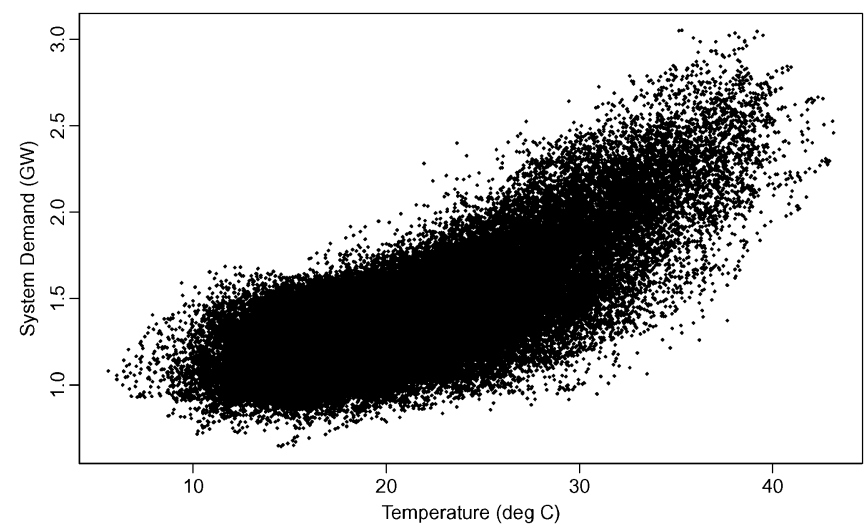

Fig. 4. Half-hourly South Australia electricity demand (excluding major industrial demand) plotted against temperature (degrees Celsius).

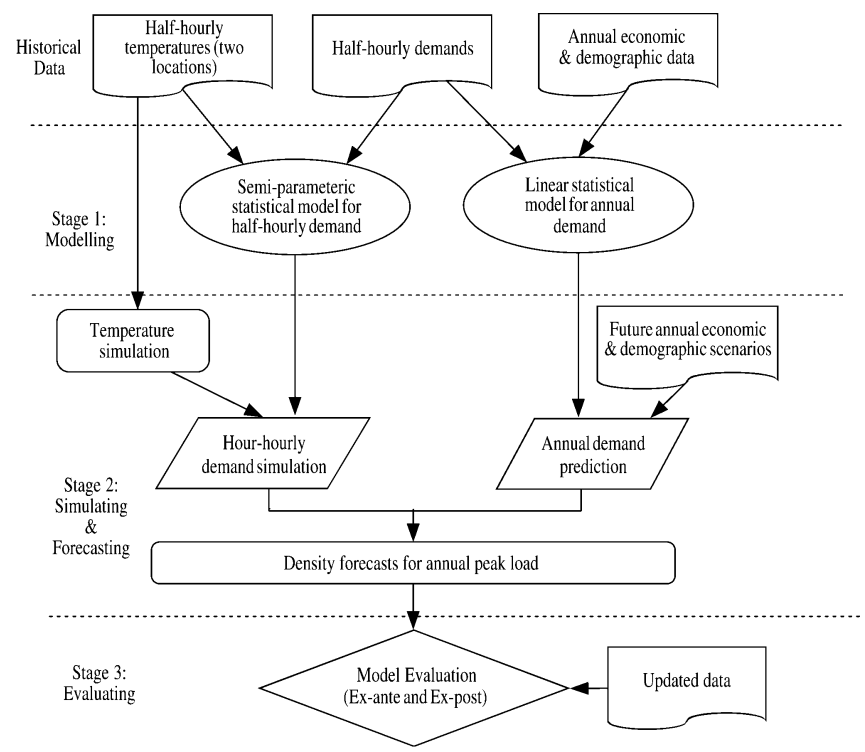

Fig. 5. Block diagram of the proposed methodology.

following annual demographic and economic observations, obtained from the Australian Bureau of Statistics (ABS) at the end of each financial year (June 30), have been considered:

1) estimated residential population;

2) persons per household;

3) number of households;

4) Adelaide CPI;

5) household sector per capita disposable income, in dollars;

6) GSP chain volume estimates, in millions of dollars;

7) average electricity price (residential and business), in cent/ $\mathrm{kWh}$;

8) air-conditioning index (the proportion of households with an air-conditioning unit).

\section{Methodology Framework}

Based on the available data described in the previous section, a new methodology to forecast density forecasts of peak load up to ten years ahead is proposed. The proposed methodological framework is illustrated in Fig. 5, and can be summarized in three stages: modeling, simulating and forecasting, and evaluating.
The relationships between demand and the driver variables, including temperatures, calendar effects and some demographic and economic variables, have been established using semi-parametric additive models [16]. The model can be split into annual effects (economic and demographic variables) and half-hourly effects (temperature and calendar variables), with the two parts of the model estimated separately. The input variables of the annual model were selected using Akaike's information criterion (AIC) and the input variables of the half-hourly model were selected by minimizing the out-of-sample forecasting errors.

In the second stage, the forecast distributions were obtained from the estimated model using a mixture of temperature and residual simulations, and future assumed demographic and economic scenarios. A new seasonal bootstrapping method with variable blocks was applied to resample residuals and temperatures. The temperature bootstrap is designed to capture the serial correlation that is present in the data due to weather systems moving across South Australia. More than 2000 years of temperatures are simulated to estimate the forecast density.

Finally, to evaluate the forecasting performance of the model, the actual demand of a summer with two different types of predictions (ex ante forecasts and ex post forecasts) were compared. Specifically, ex ante forecasts are the forecasts made in advance using whatever information is available at the time. On the other hand, ex post forecasts are those that are made using information on the "driver variables" that is only known after the event being forecast. The difference between the ex ante forecasts and ex post forecasts will provide a measure of the effectiveness of the model for forecasting (taking out the effect of the forecast errors in the input variables). The results show good forecasting capacity of the proposed model at predicting the distribution of long-term electricity demand.

The following section will explain the theory and implementation for each stage in more detail.

\section{Model Establishment}

\section{A. Model Description}

The proposed semi-parametric additive model is in the regression framework but with some nonlinear relationships and with serially correlated errors. This is similar to the models developed by others including [17] and [18], but a large number of modifications and extensions are proposed to make the models more general, robust and effective. In particular, the proposed models allow nonlinear and nonparametric terms using the framework of additive models [19].

Specific features of the models are summarized below:

- temperature effects are modeled using regression splines;

- temperatures from the last $3 \mathrm{~h}$ and the same period from the last six days are considered;

- economic and demographic variables are modeled linearly;

- errors are serially correlated.

A separate model to the data from each half-hourly period was fitted. Since the demand patterns change throughout the day, better estimates can be obtained if each half-hourly period is treated separately. This procedure of using individual models for each time of the day has also been applied by other researchers [14], [18], [20]. 
The model for each half-hour period can be written as

$$
\ln \left(y_{t, p}-o_{t, p}\right)=h_{p}(t)+f_{p}\left(\boldsymbol{w}_{1, t}, \boldsymbol{w}_{2, t}\right)+\sum_{j=1}^{J} c_{j} z_{j, t}+n_{t}
$$

where

- $y_{t, p}$ denotes the demand at time $t$ (measured in half-hourly intervals) during period $p(p=1, \ldots, 48)$;

- $o_{t, p}$ denotes the major industrial demand for time $t$ during period $p$ (here, major industrial loads are subtracted and will be modeled separately);

- $h_{p}(t)$ models all calendar effects;

- $f_{p}\left(\boldsymbol{w}_{1, t}, \boldsymbol{w}_{2, t}\right)$ models all temperature effects where $\boldsymbol{w}_{1, t}$ is a vector of recent temperatures at Kent Town and $\boldsymbol{w}_{2, t}$ is a vector of recent temperatures at Adelaide airport;

- $z_{j, t}$ is a demographic or economic variable at time $t$; its impact on demand is measured via the coefficient $c_{j}$ (these terms do not depend on the period $p$ );

- $n_{t}$ denotes the model error at time $t$.

The logarithmic demand, rather than the raw demand is modeled. A variety of transformations of demand from the Box-Cox (1964) class were tried and it was found that the logarithm resulted in the best fit to the available data. Natural logarithms have been used in all calculations. The effect of this transformation is that major industrial demand has an additive effect on demand, but calendar, temperature, economic and demographic variables have multiplicative effects on demand.

1) Calendar Effects: $h_{p}(t)$ includes handle annual, weekly and daily seasonal patterns as well as public holidays:

$$
h_{p}(t)=\alpha_{t, p}+\beta_{t, p}+\gamma_{t, p}+\delta_{t, p}+\ell_{p}(t)
$$

where

- $\alpha_{t, p}$ takes a different value for each day of the week (the "day of the week" effect);

- $\beta_{t, p}$ takes value zero on a non-work day, some non-zero value on the day before a non-work day and a different value on the day after a non-work day (the "holiday" effect);

- $\ell_{p}(t)$ is a smooth function that repeats each year (the "time of summer" effect).

The smooth function $\ell(t)$ was estimated using a cubic regression spline. Six knots were chosen at equally spaced times throughout the summer: 16.6 days, 33.6 days, 49.6 days, 66.6 days, 83.6 days, 99.6 days, 116.6 days and 132.6 days.

2) Temperature Effects: The function $f_{p}\left(\boldsymbol{w}_{1, t}, \boldsymbol{w}_{2, t}\right)$ models the effects of recent temperatures on the aggregate demand. Because the temperatures at the two locations are highly correlated, these were not used directly. Instead, the average temperature across the two sites

$$
x_{t}=\left(w_{1, t}+w_{2, t}\right) / 2
$$

and the difference in temperatures between the two sites were both used. These will be almost uncorrelated with each other making it easier to use in statistical modeling [22]. Then the temperature effects were included using the following terms:

$$
\begin{aligned}
f_{p}\left(\boldsymbol{w}_{1, t}, \boldsymbol{w}_{2, t}\right)= & \sum_{k=0}^{6}\left[f_{k, p}\left(x_{t-k}\right)+g_{k, p}\left(d_{t-k}\right)\right] \\
& +\sum_{j=1}^{6}\left[F_{j, p}\left(x_{t-48 j}\right)+G_{j, p}\left(d_{t-48 j}\right)\right] \\
& +q_{p}\left(x_{t}^{+}\right)+r_{p}\left(x_{t}^{-}\right)+s_{p}\left(\bar{x}_{t}\right)
\end{aligned}
$$

where

- $x_{t}^{+}$is the maximum of the $x_{t}$ values in the past $24 \mathrm{~h}$;

- $x_{t}^{-}$is the minimum of the $x_{t}$ values in the past $24 \mathrm{~h}$;

- $\bar{x}_{t}$ is the average temperature in the past seven days.

Each of the functions $\left(f_{k, p}, g_{j, p}, F_{k, p}, G_{j, p}, q_{p}, r_{p}\right.$ and $\left.s_{p}\right)$ was assumed to be smooth and was estimated using a cubic regression spline. Splines with knots at 22 and $29^{\circ} \mathrm{C}$ were used for $f, F$ and $q$. For the function $r$, knots at 13.8 and $16.9^{\circ} \mathrm{C}$ were used. For the function $s$, knots at 18.2 and $22.2^{\circ} \mathrm{C}$ were applied. For the functions $g$ and $G$, knots at -2.2 and $-0.7^{\circ} \mathrm{C}$ were used. In selecting the knots for the cubic regression splines, different knot positions and different numbers of knots were tried; the knot positions that provided the best forecasting performance were selected in the model.

3) Demographic and Economic Effects: The remaining terms in the model are the demographic and economic effects which enter via the summation term

$$
\sum_{j=1}^{J} c_{j} z_{j, t}
$$

Thus, each of these variables has a linear relationship with the demand coefficients $c_{1}, \ldots, c_{J}$.

Because economic relationships are usually relatively weak, and change slowly, each of these is estimated as a linear term with the same coefficient for each time period. Furthermore, the demographic and economic variables were subject to substantial measurement error. Consequently, more complicated relationships for these variables are not justified.

4) Error Term: The error term $n_{t}$ will be serially correlated, reflecting the fact that there are other environmental conditions that are not captured in this model.

\section{B. Variable Selection}

A highly significant model term does not necessarily translate into good forecasts. It is required to find the best combination of the input variables for producing accurate demand forecasts.

First, the model has been split into two separate models, one model based on the annual economic and demographic variables (the linear terms), and the other based on the remaining variables which are measured at half-hourly intervals. Thus

$$
d_{t}=\left(w_{2, t}-w_{1, t}\right)
$$

$$
\ln \left(y_{t, p}-o_{t, p}\right)=\ln \left(y_{t, p}^{*}\right)+\ln \left(\bar{y}_{i}\right)
$$



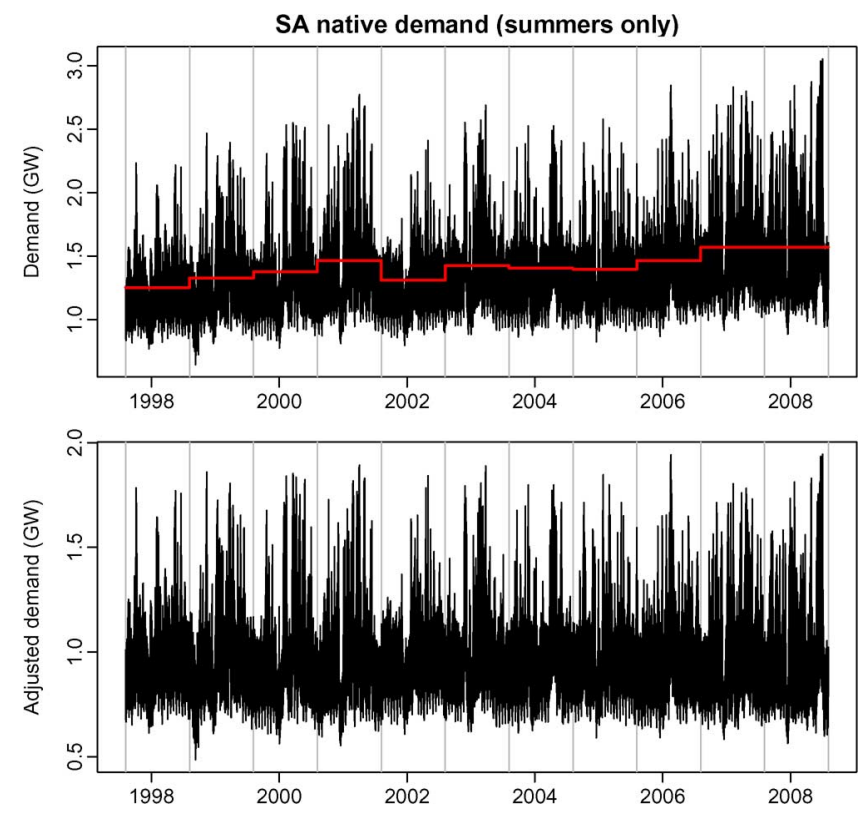

Fig. 6. Top: Half-hourly demand data for South Australia from July 1, 1997 to March 31, 2008. The median annual demand shown as a red line. Bottom: Adjusted half-hourly demand where each year of demand is adjusted to have median 1. Only data from November-March are shown.

where $\bar{y}_{i}$ is the median non-mining demand for the summer in which time period $t$ falls, and $y_{t, p}^{*}$ is the standardized nonmining demand for time $t$ and period $p$. The top panel of Fig. 6 shows the original demand data with the median annual summer values shown in red, and the bottom panel shows the half-hourly adjusted demand data. Then

$$
\ln \left(y_{t, p}^{*}\right)=h_{p}(t)+f_{p}\left(\boldsymbol{w}_{1, t}, \boldsymbol{w}_{2, t}\right)+e_{t}
$$

and

$$
\ln \left(\bar{y}_{i}\right)=\sum_{j=1}^{J} c_{j} z_{j, i}+\varepsilon_{i}
$$

where the two error terms, $e_{t}$ and $\varepsilon_{i}$, sum to $n_{t}$.

1) Temperature and Calendar Variables: A separate model of the form (5) for each half-hourly period has been fitted, i.e., 48 half-hourly demand models. For each model, the temperature and calendar variables were selected through a cross-validation procedure. That is, the data have been separated into training and validation sets, and then the input variables are selected by minimizing the accumulated prediction errors for the validation data set. Here the mean squared error (MSE) is used as the selection criterion, and only time periods between 12 noon and 8:30 pm were included in the MSE calculations, since our major concern is the peak load.

To select the input variables for the half-hourly demand model, we began with the full model including all temperature and calendar variables. The predictive value of each variable in the model was tested independently by dropping each term from the model while retaining all other terms. Omitted variables that led to a decrease in MSE were left out of the model in subsequent tests. Thus, a step-wise variable selection procedure was carried out based on out-of-sample predictive accuracy. The following input variables were selected:

- the current temperature and temperatures from the last $2.5 \mathrm{~h}$;

- temperatures from the same time period for the last two days;

- the current temperature differential and the temperature differential from the last $6 \mathrm{~h}$;

- the temperature differential from the same time period of the previous day;

- the maximum temperature in the last $24 \mathrm{~h}$;

- the minimum temperature in the last $24 \mathrm{~h}$;

- the average temperature in the last seven days;

- the day of the week;

- the holiday effect;

- the day of summer effect.

Once the best model was identified, the model was refitted using all available data.

2) Demographic and Economic Variables: Models of the form (6) were considered in selecting the various demographic and economic variables. In addition, lagged average price (due to the lagged effect of price on demand) and the number of cooling degree-days for each summer were included. For each day, the cooling degrees is defined as the difference between the mean temperature and $18.5^{\circ} \mathrm{C}$. If this difference is negative, the cooling degrees is set to zero. These values are added up for each summer to give the cooling degree-days for the summer.

Because there is so little available annual data, it is not feasible to use out-of-sample tests for variable selection with model (6). Instead, the corrected Akaike's information criterion [23, p. $202]$ is used to select the best model. The corrected AIC can be expressed as

$$
\mathrm{AIC}_{C}=-2 L+2 p\left[1+\frac{p+1}{n-p-1}\right]
$$

where $L$ is the log-likelihood of the model, $p$ is the number of parameters in the model and $n$ is the number of observations used in fitting the model. So it is a penalized likelihood method. The lower the value of the AIC, the better the model.

A large range of models were considered; the best model included the GSP, lagged average price and cooling degree days with the following estimated coefficients.

- The coefficient of GSP was $1.167 \times 10^{-5}$. That is, annual median demand increases by $e^{0.01167}-1=1.17 \%$ for every additional $\$ 1$ billion of GSP.

- The coefficient of the price variable was -0.0201 . That is, annual median demand decreases by $\left|e^{-0.0201}-1\right|=$ $1.99 \%$ for every additional cent $/ \mathrm{kWh}$ that price increases.

- The coefficient of cooling degree-days was $3.215 \times 10^{-4}$. That is, annual median demand increases by $e^{0.03215}-1=$ $3.27 \%$ for every additional 100 cooling degree days.

\section{Model Fitting}

The predictive capacity of the model has been investigated by looking at the fitted values. Fig. 7 shows the fitted annual 


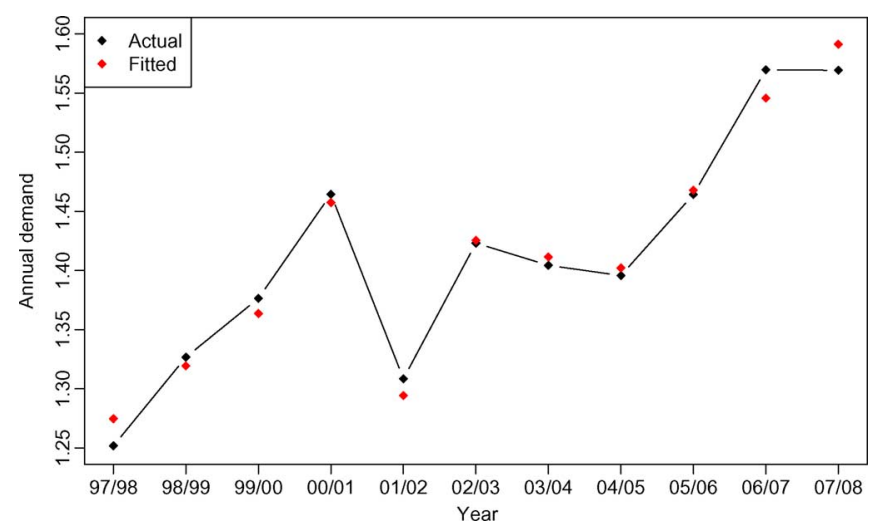

Fig. 7. Actual summer demand and summer demand predicted from the model for annual data.
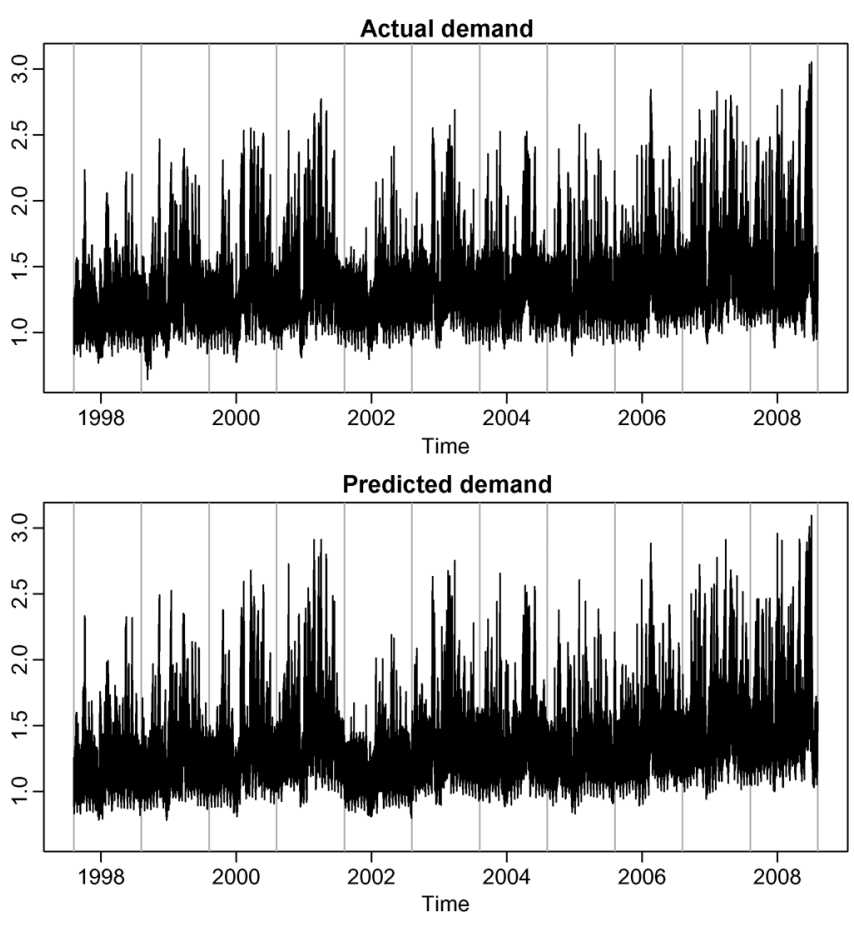

Fig. 8. Time plots of actual and predicted demand.

median demand. It can be seen that the variables have captured the underlying trend in electricity demand remarkably well.

These annual fits are combined with the fits from the halfhourly model (5), so as to obtain estimated demand at each period of historical data. These are shown in Fig. 8. Fig. 9 illustrates the model prediction for January 2008. The fitted and actual values are almost indistinguishable showing that the vast majority of the variation in the data has been accounted for through the driver variables.

These "predicted" values are not true forecasts as the demand values from these periods were used in constructing the statistical model. Consequently, they tend to be more accurate than what is possible using true forecasts.

The difference between actual demand and fitted demand are known as the residuals; these values are shown in Fig. 10. In this case, the MAE is $51.4 \mathrm{MW}$ or about $2 \%$ of the total variation in demand.

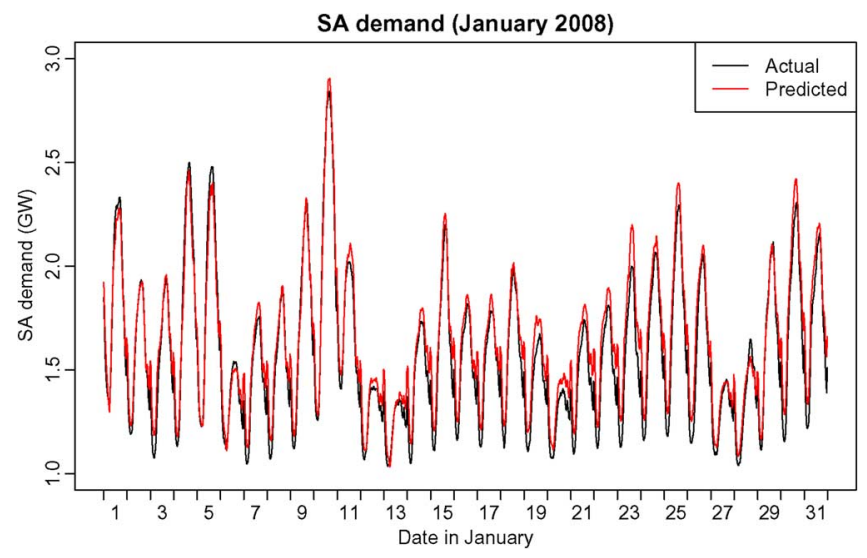

Fig. 9. Actual and predicted demand for the first three weeks of January 2008.

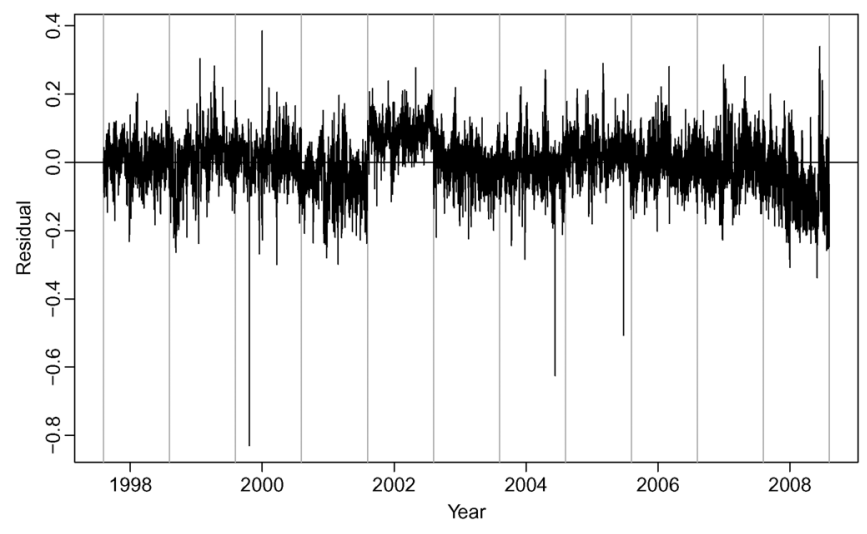

Fig. 10. Half-hourly residuals (actual-predicted) from the demand model.

The out-of-sample forecast accuracy will, on average, be worse than the in-sample forecast accuracy. In other words, the MAE calculated on out-of-sample forecasts will be higher than the MAE calculated above on in-sample predictions. In Section VI-B, out-of-sample forecast evaluation will be considered for the summer of 2007-2008.

\section{Temperature Simulation}

For forecasting, future values of the input variables are required, i.e. temperatures and economic data. The long-term GSP forecasts and pricing scenarios were obtained from AEMO.

To simulate the temperatures, a new double seasonal block bootstrap method is proposed, which involves blocks of variable length and variable start position for producing the distribution of half-hourly temperatures across the year. The effectiveness of the proposed method has been validated by reproducing the probability distribution of historical demand using simulated temperatures.

\section{A. Bootstrap Temperature Resampling}

1) Single Season Block Bootstrap: The method of bootstrapping involves randomly resampling historical data. With time series, it is important to preserve any seasonal or trend patterns as well as the inherent serial correlation. The standard method for bootstrapping time series is the "block bootstrap" [24] which involves taking random segments of the historical time series 
and pasting them together to form new artificial series. There are obviously a very large number of such series that could be formulated in this way. A key parameter in the technique is the length of each segment. This needs to be long enough to capture the essential serial correlations in the data, but short enough to allow a large number of possible simulated series to be generated.

When applied to seasonal time series, it is important that the length of each segment or block is a multiple of the length of the seasonal period. [25] calls this a "seasonal block bootstrap" although we will call it a "single season block bootstrap" to distinguish it from the double seasonal version to follow.

In a single season block bootstrap, a bootstrap series consists of whole seasons from the historical data, pasted together in a different order from how they were observed. For example, if this was applied to quarterly data from 1980-2007, then the seasonal period is four quarters or one year. A bootstrapped series of the same length as the original series would consist of a shuffled version of the data, where the data for each year stays together but the years are rearranged. For example, it may look like the following:

\begin{tabular}{|l|l|l|l|l|l|}
\hline 1999 & 1994 & 2005 & 1991 & 1986 & $\ldots$ \\
\hline
\end{tabular}

In this paper, this approach is applied to residuals from a fitted model which have a seasonal period of 48 half-hourly periods or one day. The series also show some serial correlation up to about 14 days in length. So it is reasonable to sample in blocks of 14 days, or 672 time periods. The whole series would be divided into these blocks of length 14 days. In ten years of data (each of length 151 days), there would be 107 complete blocks as in the first equation at the bottom of the page, where Block
1 consists of observations from days 1-14, Block 2 consists of observations from days 15-28, and so on.

Then a bootstrap sample is obtained by simply randomly resampling from the 107 blocks. For example, the bootstrap series may be constructed as in the second equation at the bottom of the page.

2) Double Season Block Bootstrap: The above approach is not suitable for temperature simulation because temperatures contain two types of seasonality: daily seasonality as well as annual seasonality. If the whole year was taken as the seasonal period, there would be too few years to obtain enough variation in the bootstrap samples.

Consequently, a new approach is required. The idea of [25] for time series such as half-hourly temperatures that involve two seasonal periods has been extended. Specifically, each year of data are divided into blocks of length $48 m$ where $m$ is an integer. Thus, each block is of length $m$ days. For the sake of illustration, suppose $m=$ nine days. Then block 1 consists of the first nine days of the year, block 2 consists of the next nine days, and so on. There are a total of 151/9 $=16.8$ blocks in each year. The last partial block will consist of only 7 days.

Then the bootstrap series consists of a sample of blocks 1 to 17 where each block may come from a different randomly selected year. For example, block 1 may come from 1999, block 2 from 1996, block 3 from 2003, and so on. The randomly selected years give rise to a large range of possible bootstrapped series.

The idea is best illustrated in a diagram. The original data can be arranged as in the first equation at the bottom of the next page. Then one possible bootstrap series may look like in the second equation at the bottom of the page.

The difference between this and the previous single season block bootstrap is that here, the blocks stay at the same time of

\begin{tabular}{|c|c|c|c|c|}
\hline Block 1 & Block 2 & Block 3 & Block 4 & Block 107 \\
\hline
\end{tabular}

\begin{tabular}{|l|l|l|l|}
\hline Block 105 & Block 73 & Block 39 & Block 54 $\ldots .$. Block 5 \\
\hline
\end{tabular}

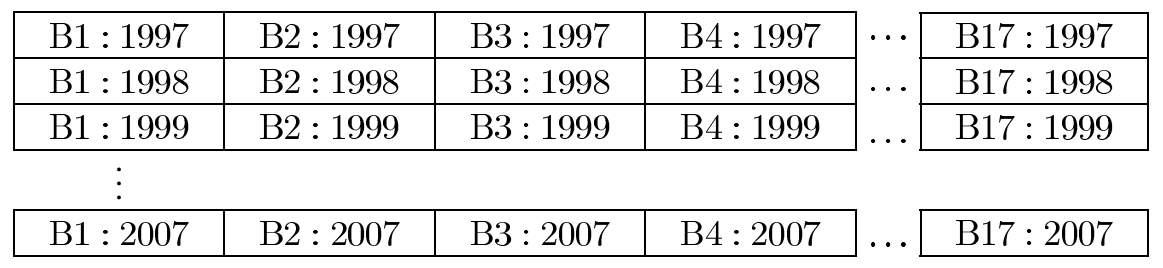


year as they were observed, although they may randomly move between years.

One problem with this double seasonal block bootstrap method is that the boundaries between blocks can introduce some unrealistic large jumps in temperature. However, this behaviour only ever occurs at midnight, thus, the phenomenon is unlikely to be a problem for the simulation purposes in this paper since we are interested in high temperature.

As explained above, the number of possible values the simulated series can take on any given day is limited to the specific historical values that have occurred on that day in previous years. With ten years of historical data, there are only ten possible values that any specific point in the simulated series can take. While calculating statistics such as probability levels for individual days, it becomes a problem as there is insufficient variation to obtain accurate estimates.

Consequently, some variation in length and position are introduced. That is, instead of having blocks of fixed length $m$, we allow the blocks to be of length between $m-\Delta$ days and $m+\Delta$ days where $0 \leq \Delta<m$. Further, instead of requiring blocks to remain in exactly the same location within the year, we allow them to move up to $\Delta$ days from their original position. This has little effect on the overall time series patterns provided $\Delta$ is relatively small, and allows each temperature in the simulated series to take a greater number of different values.

A uniform distribution is used on $(m-\Delta, m+\Delta)$ to select block length, and an independent uniform distribution on $(-\Delta, \Delta)$ to select the variation on the starting position for each block.

This "double seasonal block bootstrap with variable blocks" performs much better in producing the distribution of temperatures on a given day due to the larger number of potential values the simulated temperatures can take.

The simulated temperatures are actually adjusted slightly by adding some additional noise to allow the distribution of daily maximum temperatures to closely match those observed since 1900. The same noise value is added to each time period in a block and to both temperature sites. This ensures the serial correlations and the cross-correlations are preserved.

\section{B. Probability Distribution Reproduction}

To validate the effectiveness of the proposed temperature simulation, the probability distribution of historical demand is reproduced using known economic values but simulating temperatures, and then compare with the real demand data.

Fig. 11 shows PoE levels for all years of historical data using known economic values but simulating temperatures. These are

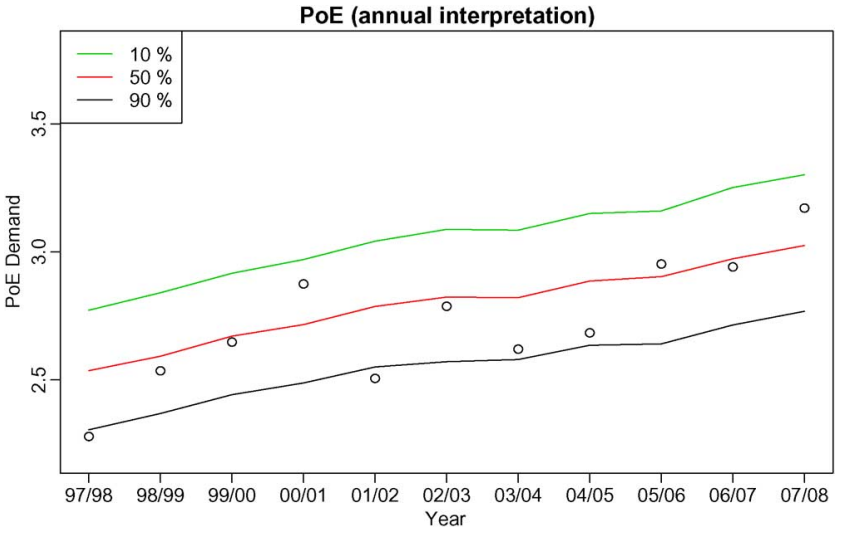

Fig. 11. PoE levels for all years of historical data using known economic values but simulating temperatures.

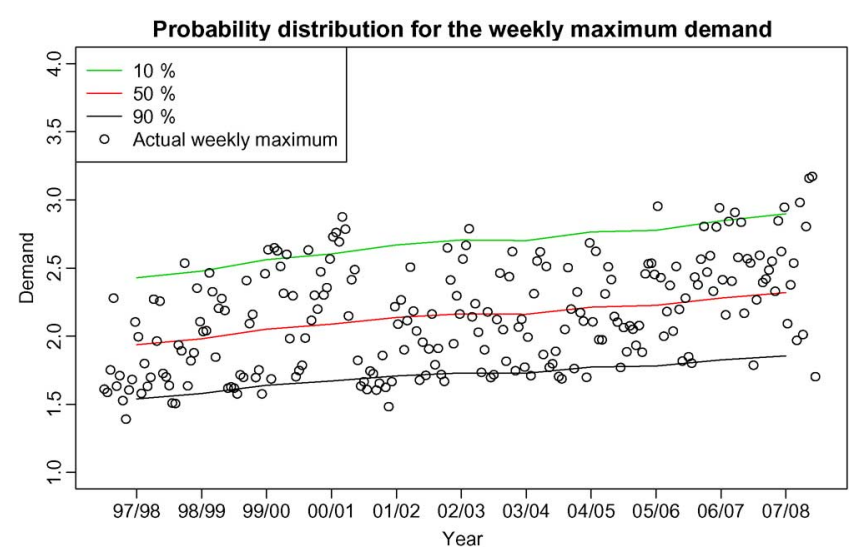

Fig. 12. Probability distribution of the weekly maximum demand for all years of historical data using known economic values but simulating temperatures.

based on the distribution of the annual maximum in each year. Fig. 12 shows similar levels for the weekly maximum demand. There are three annual maximums above the 50\% PoE level, two annual maximums below the $90 \%$ PoE level and no annual maximums above the $10 \%$ PoE levels. Of the 231 historical weekly maximum demand values in the past eleven summers, there have been nineteen above the $10 \%$ distribution level and twenty-one below the $90 \%$ distribution level. Therefore, it can be inferred that the reproduced probability distribution are within the ranges that would occur by chance with high probability (based on a binomial distribution). This provides good evidence that the forecast distributions will also be reliable for future years.

\begin{tabular}{|c|c|c|c|l|l|}
\hline B1 $: 2007$ & B2 $: 2005$ & B3 $: 2002$ & B $4: 2001$ & $\cdots$ & B17 $: 1999$ \\
\hline B1 $: 1999$ & B2 $: 2007$ & B3 $: 2002$ & B $4: 1999$ & $\cdots$ & B17 $: 1998$ \\
\hline B1 $: 2002$ & B2 $: 1997$ & B3 $: 2003$ & B $4: 2001$ & $\cdots$ & B17 $: 2004$ \\
\hline$\vdots$ & $\cdots$ \\
\hline B1 $: 2003$ & B2 $: 2003$ & B3 $: 2004$ & B $4: 2006$ & $\ldots$ & B17 $: 1997$ \\
\hline
\end{tabular}



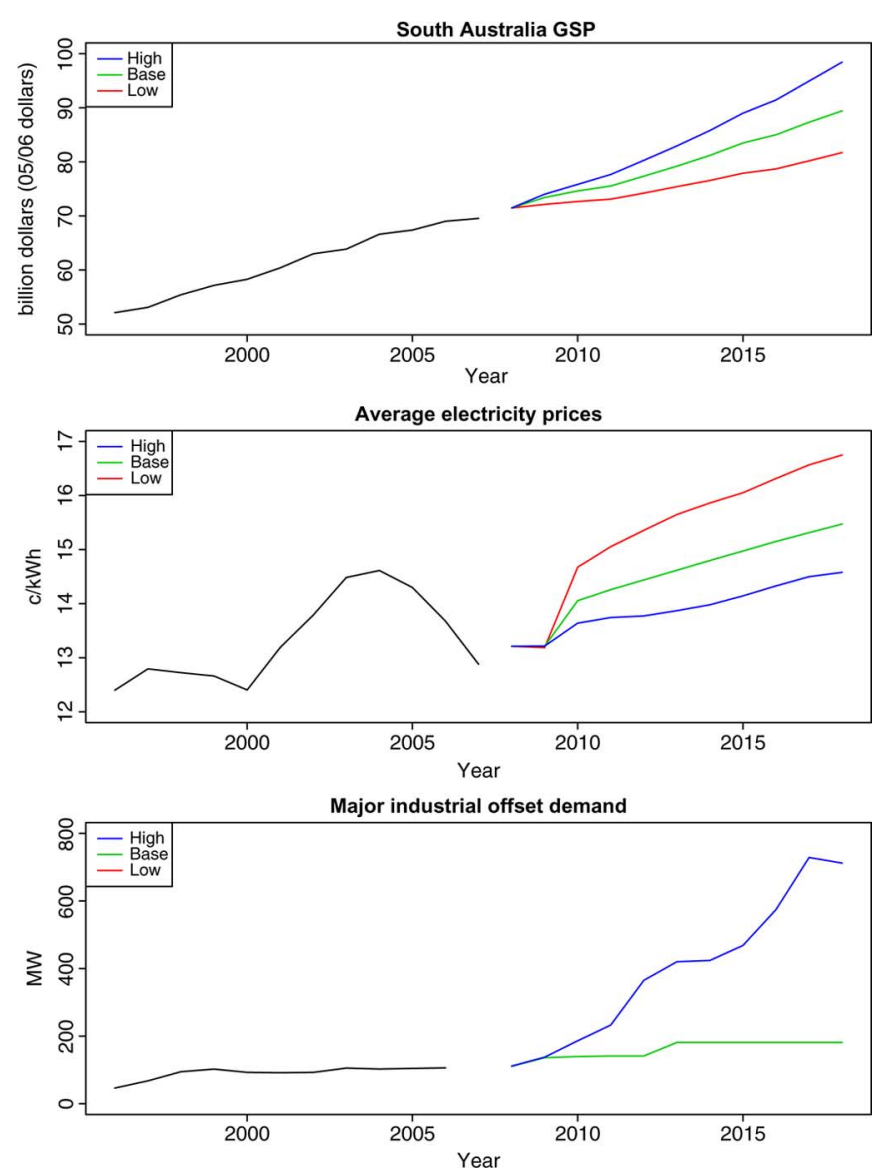

Fig. 13. Three future scenarios for major industrial demand and South Australia's GSP and average electricity price. The bottom panel shows the predicted effect on annual median demand for South Australia.

\section{FORECASTING AND EVALUATION}

The distribution of annual and weekly peak electricity demand are forecasted up to ten years ahead, and evaluate the model performance by comparing the forecasting results with the real demand data for summer 2007-2008.

\section{A. Forecasting Results}

Forecasts of the distribution of demand are computed by simulation from the fitted model as described above. The temperatures at two locations are simulated from historical values observed in 1997-2008 using the proposed double seasonal bootstrap with variable length. A total of 2000 years of temperature profiles were generated in this way for each year to be forecast.

Three different future scenarios for GSP, major industrial loads and average retail price were provided by AEMO and are shown in Fig. 13. Future major industrial loads have been set based on the expected level of activity at these sites.

The simulated temperatures, known calendar effects, assumed values of GSP, electricity price and major industrial demand, and simulated residuals, are all combined using the fitted statistical model to give simulated electricity demand for every half-hourly period in the years to be forecast. Thus, what could happen in the years 2008-2018 are being predicted
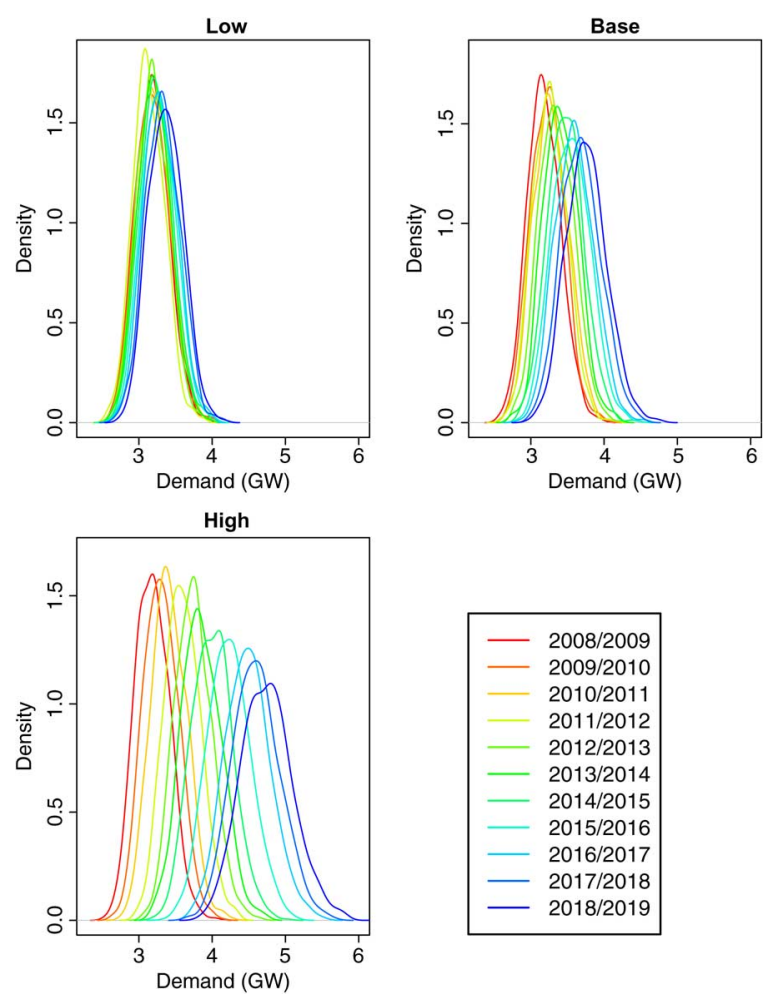

Fig. 14. Distribution of simulated annual maximum demand for 2008-2009 to 2018-2019.

under these assumed economic and demographic forecasting scenarios, but allowing for random variation in temperature events and other conditions.

To simulate the major industrial demand, $o_{t, p}$, it is assumed that it takes the form $o_{t, p}=\bar{o}_{t}+u_{t, p}$ where $\bar{o}_{t}$ is the annual major industrial demand and $u_{t, p}$ is a (serially correlated) residual term that is independent of all other terms in the model. Another single seasonal block bootstrap of length 20 days is used to simulate $u_{t, p}$ and assume $\bar{o}_{t}$ is equal to the specified values in Fig. 13 (the low and base values are identical).

1) Probability Distributions: The forecast distributions of the weekly maximum half-hourly demand are calculated for any randomly chosen week in summer, and the annual maximum half-hourly demand, using kernel density estimation [26]. Fig. 14 shows the simulated annual maximum demand densities for 2008-2009 to 2018-2019. Similarly, Fig. 15 shows the simulated weekly maximum demand densities for 2008-2009 to 2018-2019.

2) Probability of Exceedance: "Probability of exceedance" levels are also calculated from the simulated data. Suppose we are interested in the level $x$ such that the probability of the annual maximum demand exceeding $x$ is $p$. Then $x$ is the $(1-$ $p$ )th quantile of the distribution of the simulated demand values, which can be computed using the approach recommended by [27].

PoE values based on the annual maxima are plotted in Fig. 16, along with the historical PoE values and the observed annual maximum demand values. Fig. 17 shows similar levels for the 

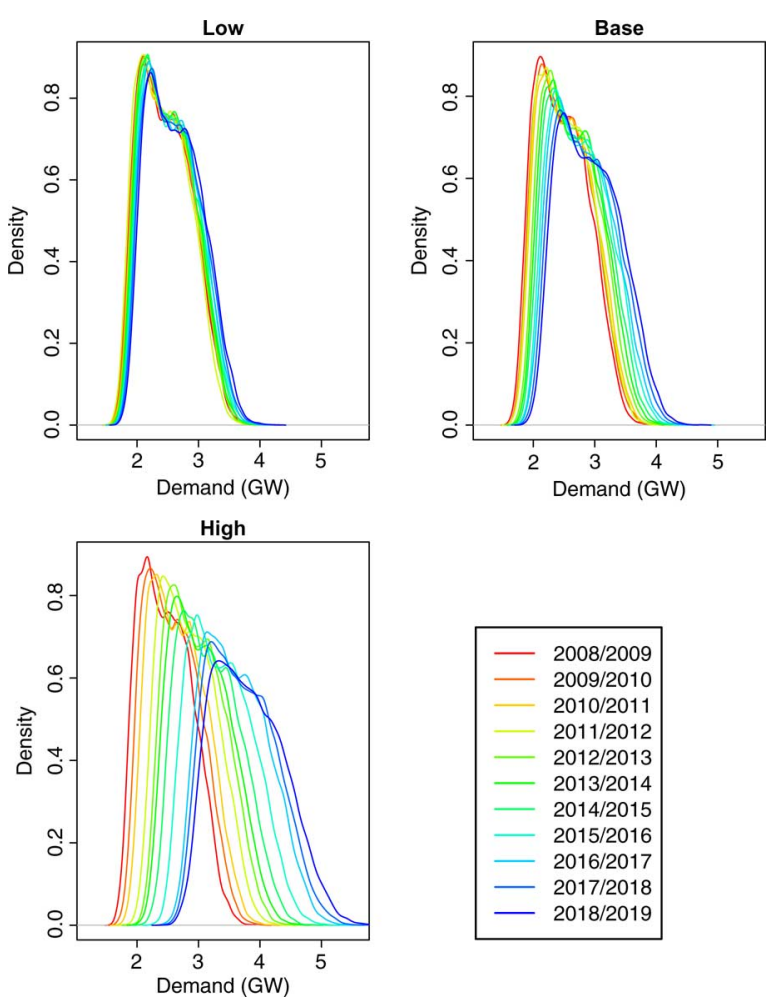

Fig. 15. Distribution of simulated weekly maximum demand for 2008-2009 to 2018-2019.

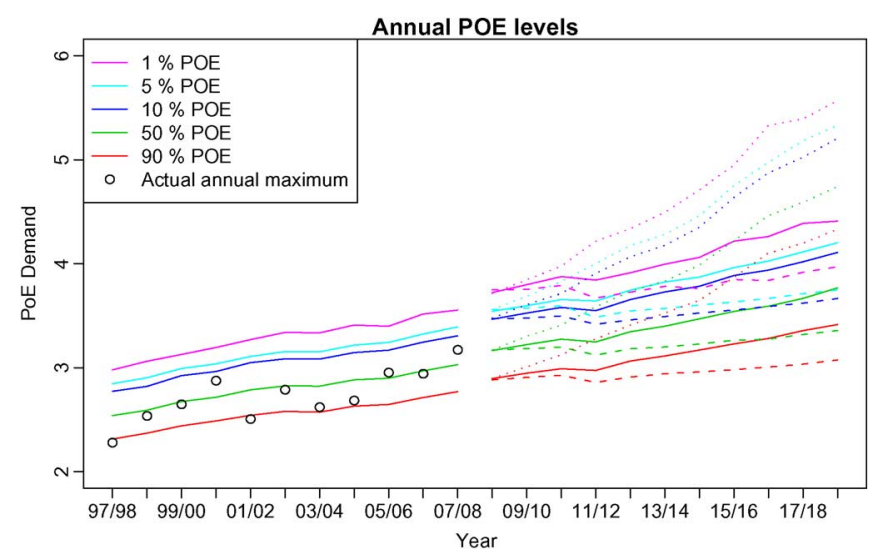

Fig. 16. Probability of exceedance values for past and future years. High scenario: dotted lines. Base scenario: solid lines. Low scenario: dashed lines.

weekly maximum demand where $p$ is the probability of exceedance in a single year.

\section{B. Model Evaluation}

To evaluate the forecasting performance, the actual demand of the last summer of data (2007-2008) has been compared with two different types of predictions: ex ante forecasts and ex post forecasts. Specifically, ex ante forecasts are those that are made using only the information that is available in advance; consequently, 2007-2008 summer demands are calculated using economic conditions as assumed in 2007 and simulated temperatures based on models for data up to 2007.

On the other hand, ex post forecasts are those that are made using known information on the "driver variables". In this eval-

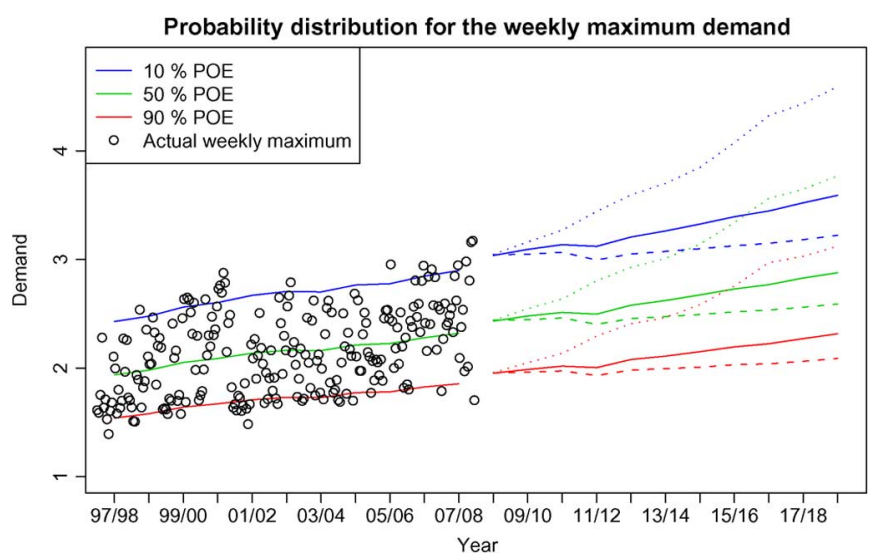

Fig. 17. Probability distribution levels for past and future years. High scenario: dotted lines. Base scenario: solid lines. Low scenario: dashed lines.

uation, ex post forecasts for 2007-2008 summer are calculated using known economic conditions in 2007 and known temperatures for the summer of 2007-2008. Data from the forecast period are not used for the model estimation or variable selection. Ex post forecasts can assume knowledge of the input or driver variables, but should not assume knowledge of the data that are to be forecast.

The difference between the ex ante forecasts and ex post forecasts will provide a measure of the effectiveness of the model for forecasting (taking out the effect of the forecast errors in the input variables).

Fig. 18 illustrates the ex ante forecast density function for maximum weekly demand and maximum annual demand for 2007-2008.

These graphs demonstrate that the actual demand values fit the ex ante forecast distributions remarkably well. The upper graph provides the best evidence of the performance of the model. In this case, the 21 actual weekly maximum demand values all fall within the region predicted from the ex ante forecast distribution. Although there is only one annual maximum demand observed, the bottom graph shows that this also falls well within the predicted region. Fig. 18 also indicates the effect of updating and including the ex post economic information subject to the use of simulated temperatures and random components. The graphs also show that the uncertainty associated with the economic variables is very small compared to the uncertainty due to temperatures and other sources of variation. However, there is considerable uncertainty associated with the economic forecasts themselves, especially over the longer term, since economic relationships might change over time (e.g., uncertainties about the impact of carbon pricing, adoption of new efficiency measures and new technologies).

All the numerical studies have been performed using R [28], running on a PC with 4 GB of RAM and $2.33-\mathrm{GHz}$ clock frequency (duo core CPU). The running time, including training and forecasting, is about one hour when simulating 2000 years of temperatures.

\section{CONCLUSION}

The major contribution of this paper is to propose a new and systematic methodology to forecast the density of long-term 


\section{ACKNOWLEDGMENT}

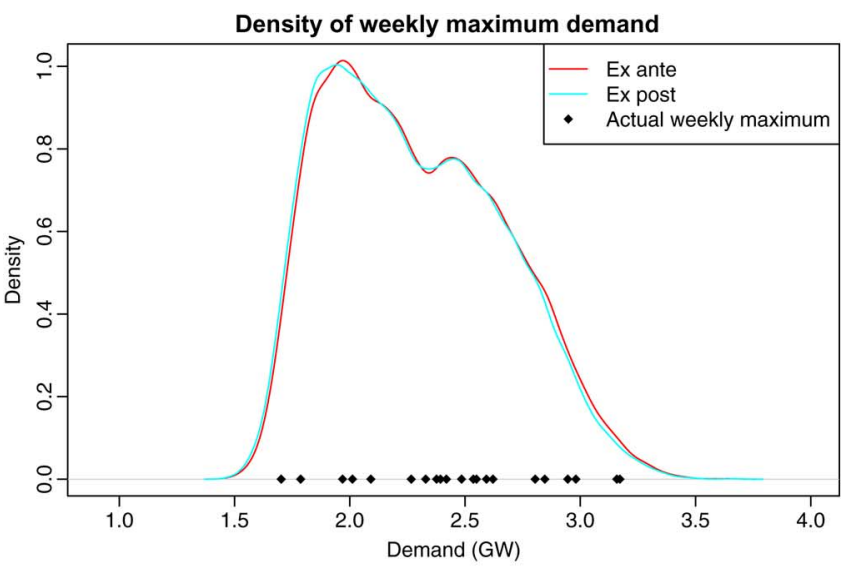

Density of annual maximum demand

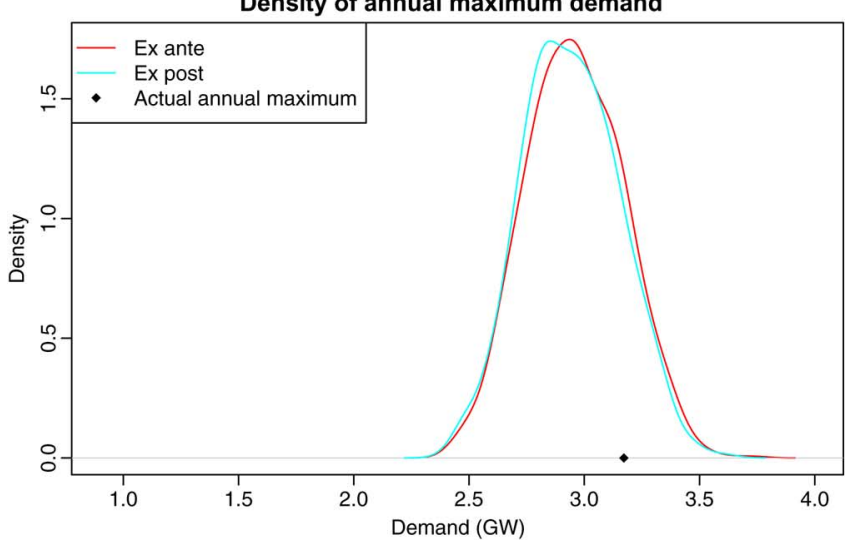

Fig. 18. Ex ante probability density functions for half-hourly, weekly maximum demand and annual maximum demand. Actual values and ex post forecasts are also shown.

peak electricity demand, including modeling, simulating and forecasting. The key features of the proposed methodology are that it provides a full density forecast for the peak demand several years ahead, with quantifiable probabilistic uncertainty. The demand model is split into annual effects and half-hourly effects, with the two parts of the model estimated separately. A new seasonal bootstrapping method with variable blocks has been implemented to simulate the temperatures. The proposed methodology captures the complex nonlinear effect of temperature and allows for calendar effects, price changes, economic growth, and other possible drivers. The forecasting results demonstrate that the model performs remarkably well on the historical data.

As with any new methodology, there are some areas for possible future improvement. For instance, the long-term temperature patterns are assumed to be stable in this work; however, climate change is likely to have much more complex and subtle effects on future temperature patterns and distributions. We are endeavouring to incorporate some long-term climate change predictions into the model. Another area for development is to increase the number of temperature sites in the model. For South Australia's geographically concentrated population, two temperature sites may be adequate. But for other regions, a larger number of temperature sites would be desirable.
The authors would like to thank the Australian Energy Market Operator (AEMO) for supplying the data used in this paper. They also would like to thank G. Richards and D. Swift of AEMO for their valuable comments and feedbacks on this research work, and the referees for their valuable comments and suggestions.

\section{REFERENCES}

[1] Power Systems Planning and Development, Load Forecasting White Paper, National Electricity Market Management Company Limited, 2005, Tech. Rep.

[2] R. Weron, Modeling and Forecasting Electricity Loads and Prices: A Statistical Approach. New York: Wiley Finance, 2006.

[3] S. J. Huang and K. R. Shih, "Short-term load forecasting via ARMA model identification including non-Gaussian process considerations," IEEE Trans. Power Syst., vol. 18, no. 2, pp. 673-679, May 2003.

[4] J. W. Taylor, "Short-term load electricity demand forecasting using double seasonal exponential smoothing," J. Oper. Res. Soc., vol. 54, pp. 799-805, 2003.

[5] H. M. Al-Hamadi and S. A. Soliman, "Long-term/mid-term electric load forecasting based on short-term correlation and annual growth," Elect. Power Syst. Res., vol. 74, pp. 353-361, 2005.

[6] P. E. McSharry, S. Bouwman, and G. Bloemhof, "Probabilistic forecast of the magnitude and timing of peak electricity demand," IEEE Trans. Power Syst., vol. 20, no. 2, pp. 1166-1172, May 2005.

[7] B. Kermanshahi, "Recurrent neural network for forecasting next 10 years loads of nine Japanese utilities," Neurocomputing, vol. 23, pp. 125-133, 1998

[8] H. Hippert, C. Pedreira, and R. Souza, "Neural networks for short-term load forecasting: A review and evaluation," IEEE Trans. Power Syst., vol. 16, no. 1, pp. 44-55, Feb. 2001.

[9] N. Amjady, "Day-ahead price forecasting of electricity markets by a new fuzzy neural network," IEEE Trans. Power Syst., vol. 21, no. 2, pp. 887-896, May 2006.

[10] N. Amjady, "Short-term bus load forecasting of power systems by a new hybrid method," IEEE Trans. Power Syst., vol. 22, no. 1, pp. 333-341, Feb. 2007.

[11] M. S. Kandil, S. M. El-Debeiky, and N. E. Hasanien, "Long-term load forecasting for fast developing utility using a knowledge-based expert system," IEEE Trans. Power Syst., vol. 17, no. 2, pp. 491-496, May 2002.

[12] K. B. Song, Y. S. Baek, D. H. Hong, and G. Jang, "Short-term load forecasting for the holidays using fuzzy linear regression method," IEEE Trans. Power Syst., vol. 20, no. 1, pp. 96-101, Feb. 2005.

[13] B. J. Chen, M. W. Chang, and C.-J. Lin, "Load forecasting using support vector machines: A study on EUNITE competition 2001," IEEE Trans. Power Syst., vol. 19, no. 4, pp. 1821-1830, Nov. 2004.

[14] S. Fan and L. Chen, "Short-term load forecasting based on an adaptive hybrid method," IEEE Trans. Power Syst., vol. 21, no. 1, pp. 392-401, Feb. 2006.

[15] H. Morita, T. Kase, Y. Tamura, and S. Iwamoto, "Interval prediction of annual maximum demand using grey dynamic model," Int. J. Elect. Power Energy Syst., vol. 18, no. 7, pp. 409-413, 1996.

[16] D. Ruppert, M. P. Wand, and R. J. Carroll, Semiparametric Regression. New York: Cambridge Univ. Press, 2003.

[17] P. E. McSharry, S. Bouwman, and G. Bloemhof, "Probabilistic forecasts of the magnitude and timing of peak electricity demand," IEEE Trans. Power Syst., vol. 20, no. 2, pp. 1166-1172, May 2005.

[18] R. Ramanathan, R. F. Engle, C. W. J. Granger, F. Vahid-Araghi, and C. Brace, "Short-run forecasts of electricity loads and peaks," Int. J. Forecast., vol. 13, pp. 161-174, 1997.

[19] T. Hastie and R. Tibshirani, Generalized Additive Models. London, U.K.: Chapman \& Hall/CRC, 1990.

[20] D. Fay, J. V. Ringwood, M. Condon, and M. Kelly, "24-h electrical load data-A sequential or partitioned time series?," Neurocomputing, vol. 55, no. 3-4, pp. 469-498, 2003.

[21] G. E. P. Box and D. R. Cox, "An analysis of transformations," J. R. Statist. Soc., Series B, vol. 26, no. 2, pp. 211-252, 1964.

[22] B. Mizrach, Forecast Comparison in $L_{2}$ Rutgers Univ., Dept. Econ., Departmental Working Papers 1995-24, 1995. [Online]. Available: $\mathrm{ftp} / / /$ snde.rutgers.edu/Rutgers/wp/1995-24.pdf. 
[23] F. E. Harrell, Jr., Regression Modelling Strategies: With Applications to Linear Models, Logistic Regression, and Survival Analysis. New York: Springer, 2001.

[24] D. N. Politis, "The impact of bootstrap methods on time series analysis," Statist. Sci., vol. 18, no. 2, pp. 219-230, 2003.

[25] D. N. Politis, "Resampling time series with seasonal components," in Frontiers in Data Mining and Bioinformatics: Proc. 33rd Symp. Interface of Computing Science and Statistics, 2001, pp. 619-621.

[26] B. W. Silverman, Density Estimation for Statistics and Data Analysis. London, U.K.: Chapman \& Hall, 1986.

[27] R. J. Hyndman and Y. Fan, "Sample quantiles in statistical packages," Amer. Statistician, vol. 50, pp. 361-365, 1996.

[28] R Development Core Team, R: A Language and Environment for Statistical Computing R Foundation for Statistical Computing. Vienna, Austria, 2009. [Online]. Available: http://www.R-project.org.

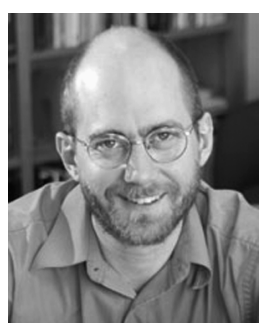

Rob J. Hyndman received the B.Sc. (Hons.) degree and the Ph.D. degree in 1993 from the University of Melbourne, Parkville, Australia.

$\mathrm{He}$ is a Professor of statistics and Director of the Business and Economic Forecasting Unit at Monash University, Clayton, Australia. He is the author of over 100 research papers and ten books. His research interests include statistical forecasting, nonparametric modeling, and computational statistics.

Dr. Hyndman is the Editor-in-Chief of the International Journal of Forecasting. He received the 2007 Moran Medal from the Australian Academy of Science for his contributions to statistical research.

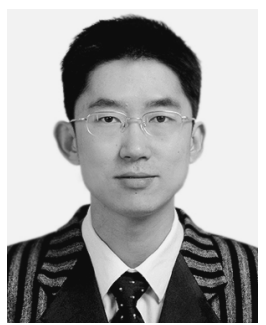

Shu Fan (M'08) received the B.S., M.S., and Ph.D. degrees in the Department of Electrical Engineering of Huazhong University of Science and Technology (HUST), Wuhan, China, in 1995, 2000, and 2004, respectively.

He conducted postdoctoral research sponsored by the Japanese Government in Osaka Sangyo University from 2004 to 2006. He was a Visiting Assistant Professor at the Energy Systems Research Center at the University of Texas at Arlington from 2006 to 2007. Presently, he is a Senior Research Fellow at Monash University, Clayton, Australia. His research interests include energy system forecasting, power system control, and high-power power electronics. 